\title{
Expression of basal cell marker revealed by RAM11 antibody during epithelial regeneration in rabbits
}

\author{
Grzegorz J. Lis ${ }^{1}$, Ewa Jasek ${ }^{1}$, Jan A. Litwin'1, Mariusz Gajda ${ }^{1}$, Joanna Zarzecka ${ }^{2}$, \\ Tadeusz Cichocki ${ }^{1}$
}

\author{
${ }^{1}$ Department of Histology; ${ }^{2}$ Department of Conservative Dentistry and Endodontics, \\ Institute of Stomatology, Jagiellonian University Medical College, Kraków, Poland
}

\begin{abstract}
RAM11 is a mouse monoclonal anti-rabbit macrophage antibody recognizing connective tissue and vascular macrophages. Our previous report showed that RAM11 reacted with basal cells of stratified squamous epithelia of rabbit skin, oral mucosa and esophagus. The aim of the present study was to follow the appearance of RAM11 immunoreactivity in basal cells of regenerating oral epithelium in rabbits. No RAM11 immunostaining was observed in the regenerating epithelium examined on days 1 and 3 of wound healing. A weak immunofluorescence first appeared on day 7 in single basal cells and $32 \%$ of RAM11- positive basal cells were observed on day 14 . These findings indicate that expression of the antigen recognized by RAM11 antibody is a transient event in the differentiation of oral keratinocytes which not always occurs during epithelial repair, although it is a constant feature of epithelial turnover in mature epithelium. Therefore this antigen can be regarded as basal cell marker only in mature stratified squamous epithelia.
\end{abstract}

Key words: RAM11, macrophages, oral epithelium, wound healing, rabbit

\section{Introduction}

RAM11 is a monoclonal $\operatorname{IgG}_{1}$ antibody developed by immunization of mice with the rabbit alveolar macrophage extract. The antibody binds a cytoplasmic, so far unknown antigen and has been used for identification of macrophages in rabbit tissues. It does not crossreact with human, rat and monkey macrophages [1].

RAM11 has been widely used for immunohistochemical demonstration of rabbit macrophages, particularly in the cellular analysis of the atherosclerotic lesions as well as in morphological analysis of normal rabbit arteries [1,2]. RAM11 immunoreactivity was also described in macrophages of injured skeletal or cardiac muscle and ocular uvea [3-5]. We previously demonstrated that RAM11 reacted also with basal cells of stratified squamous epithelia of rabbit skin, oral mucosa and esophagus [6]. This suggests the presence of a common epitope in both rabbit macrophages and basal epithelial cells - either incidental or indicating some kind of macrophage-basal cell phenotypic and functional association.

Correspondence: G.J. Lis, Dept. of Histology, Jagiellonian University Medical College, Kopernika 7, 31-034 Krakow, Poland; fax./tel.: (+4812) 4227027, e-mail: mmlis@cyf-kr.edu.pl
During the reparative processes, there is a substantial increase in the proliferative activity of basal layer cells. It seemed therefore interesting to investigate expression of the antigen recognized by RAM11 in the regenerating epithelium and to check its possible association with the processes of proliferation and/or early differentiation of the epithelial cells.

\section{Materials and methods}

We used material collected during a larger study on rabbit oral wound regeneration. All animal procedures were approved by the Jagiellonian University Ethical Committee on Animal Experiments. Six $\mu \mathrm{m}$ thick paraffin sections were prepared from the whole thickness wounds (area $1 \times 1 \mathrm{~cm}$ ) surgically created in rabbits (New Zeland White), and excised with the inact margin in the course of spontaneous healing on days 1, 3, 7 and 14. Some sections were stained routinely with HE, the others were used for immunohistochemistry. After preincubation with 5\% normal goat serum for 40 min at room temperature, sections were incubated overnight with primary antibodies (Table 1) in a humid chamber at room temperature followed by goat anti-mouse Cy-3-conjugated antibodies (Jackson IR, West Grove, PA, USA, code No. 115-165-146; dilution 1:400) applied for $90 \mathrm{~min}$. Cell nuclei were counterstained with DAPI (Sigma, Saint Louis, MO, USA). Sections were mounted in glycerol/PBS solution $(\mathrm{pH}=8.6)$. Negative controls were performed by omitting the primary antibodies during the first incubation. Rabbit spleen and human skin malignant melanoma were used as positive controls for RAM11 and S100 protein, respectively. 
Table 1. Primary antibodies used in the study.

\begin{tabular}{|l|l|c|c|}
\hline \multicolumn{1}{|c|}{ Monoclonal antibodies } & \multicolumn{1}{c|}{ Supplier } & Code & Dilution \\
\hline Mouse anti-rabbit macrophage (RAM11) & Dako, Glosrup, Denmark & M0633 & $1: 100$ \\
\hline Mouse Anti-S100 protein & Chemicon, Temecula, CA, USA & MAB079-1 & $1: 200$ \\
\hline Mouse anti-PCNA & Chemicon, Temecula, CA, USA & MAB424 & $1: 300$ \\
\hline
\end{tabular}

Sections were examined under Olympus BX50 light/fluorescence microscope. Images were collected using DP-71 digital CCD camera (Olympus, Japan) and IBM PC-class computer equipped with analySIS-FIVE ${ }^{\circledR}$ (Soft Imaging System $\mathrm{GmbH}$, Münster, Germany) image analysis system. The quantity of RAM11-immunopositive basal cells was expressed as the percentage of the total number of basal cells in regenerating epithelium present in the plane of wound section.

\section{Results}

In normal oral mucosal epithelium, RAM11 immunoreactivity was revealed by almost all cells of the basal layer. The signal was seen as small fluorescent cytoplasmic granules, sometimes preferentially located in the supranuclear region.

In regenerating epithelium examined on days 1 and 3 of wound healing, no RAM11-positive cells were observed (Fig. 1). A weak immunofluorescence first appeared on day 7 in single basal cells, located at a variable distance from the wound margin. On day $14,32 \%$ of the basal cells in the regenerating epithelium were RAM11-positive and the intensity of immunofluorescence was lower than in the intact epithelium (Fig. 2). The distribution of RAM11-positive cells was not uniform along the regenerating epithelium: there were areas with only single immunoreactive cells and areas where almost all cells showed mild to moderate RAM11 expression. In the latter areas, basal cells were cuboidal/columnar (stationary), whereas in areas containing elongated (migratory) basal cells, they were mostly RAM11 negative (Fig. 3). Generally, RAM11 was expressed earlier in cells located closer to the intact epithelium, although this was not a consistent finding. Immunoreactivity in the interpapillary zone appeared stronger than on the papillary tips what corresponded with the pattern observed in normal epithelium. However, in the regenerating oral mucosa, papillae were very irregular and limited in number.

Nuclear expression of PCNA was present in almost all basal and parabasal cells in both, intact and regenerating epithelium (Fig. 4) at all studied stages of the reparative process.

The immunostaining for S100 protein subunit A was occasionally found in some cells of intermediate layer, but only in the intact epithelium. The basal cells were always negative (Fig. 5).

\section{Discussion}

It is difficult to interpret observations presented in this study as long as the nature of antigen recognized by RAM11 antibody is not clarified. It is not associated with melanocytic differentiation, as proven by the absence of S100 immunostaining in the regenerating epithelium. RAM11-positive cells have never been observed in parabasal and intermediate layers of the epithelium, where Langerhans cells are located. This means that RAM11 labels oral keratinocytes.

The basal cell layer in oral epithelium is a heterogeneous population of proliferating and differentiating oral keratinocytes including epithelial stem cells, transit amplifying cells and postmitotic differentiating cells. The epithelial stem cells are crucial for replenishment of the wounded epithelium, while their direct progeny, transient amplifying cells with high proliferative potential, are committed to generate postmitotic diferentiating cells which undergo terminal differentiation [7-10].

During epithelial regeneration, expression of basal cell cytoplasmic antigen revealed by RAM11 antibody is delayed: it first appears on post-wound day 7 and even on day 14 only one-third of the basal cells are

Figs. 1-5. Fluorescence microscopy images of regenerating epithelium (RAM11, PCNA, S-100: red channel images; DAPI: blue channel image). Positive signals in the connective tissue represent of erythrocytes (autofluorescence, RAM11) and nerve fibers (S-100). Fig. 1. Regenerating epithelium on day 3, close to wound margin (arrowhead). No RAM11 immunostaining in the basal layer (base of epithelium arrowed). Fig. 2. Intact epithelium (left) and regenerating epithelium on day 14. Note intense RAM11 immunofluorescence in the basal layer of intact epithelium and weaker immunoflurescence of some basal cells of the regenerating epithelium located at different distances from the wound margin (some of them arrowed). Fig. 3. Regenerating epithelium on day 14 showing areas with RAM11-positive (mostly cuboidal/columnar) and RAM11-negative (mostly elongated) basal cells. Inset: framed area showing DAPI stained cell nuclei. Note cuboidal/columnar shape of basal cells in RAM11 positive areas as indicated by the arrangement of the nuclei. Fig. 4. PCNA in regenerating epithelium on day 14. Almost all basal and parabasal cells show nuclear expression of the antigen. Fig. 5. Negative immunoreactivity for S100 protein in regenerating epithelium on day 14. All bars: $50 \mu \mathrm{m}$. 

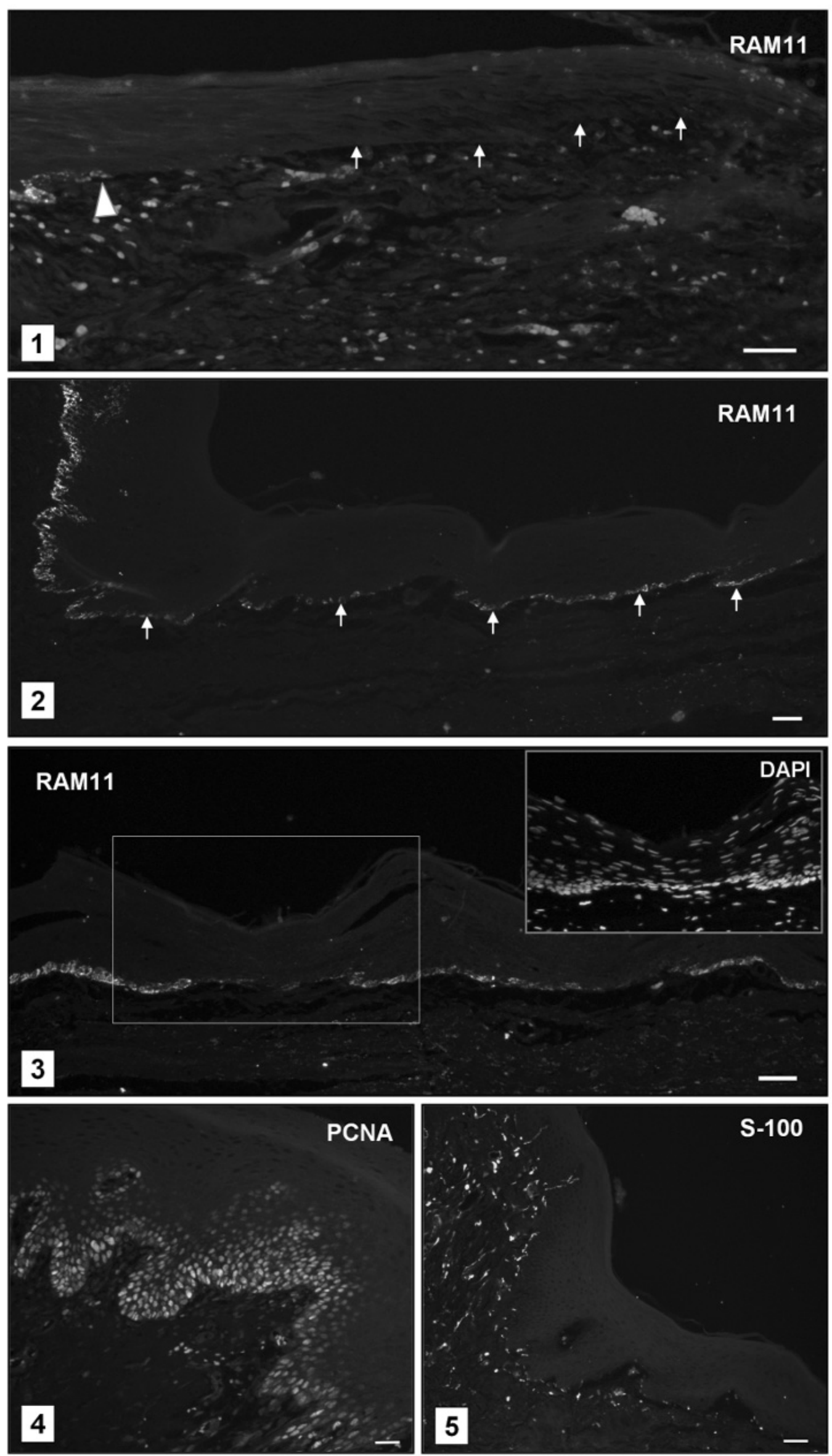

CPolish Histochemical et Cytochemical Society

Folia Histochem Cytobiol. 2010:48(1): 91 (89-92)

10.2478/v10042-008-0087-3 
RAM11-positive. While practically all basal cells in the intact epithelium are RAM11-positive, this observation shows that RAM11 labels an antigen whose expression is suppressed during early repair process. Furthermore, we found that the earliest, weak expression of this antigen was revealed by single basal cells located at a variable distance from the margin of the wound. This may suggest that expression of the epitope recognized by RAM11 antibody is not strictly related to the regeneration sequence ("age" of cells). Similar observation was made in case of heat shock protein (Hsp) expression in mouse skin during wound healing. Changes in Hsp expression pattern were not related to the time after wounding, being rather dependent on the locations of keratinocytes in particular layers of the regenerating epidermis [11].

The nuclear expression of PCNA was observed in all basal and parabasal cells in the regenerating epithelium at all studied stages of the reparative process, what indicates that RAM11 immunoreactivity with its delayed and limited expression is not related to proliferative activity of basal cells. Stem cells are preferentially located at the tips of dermal papillae [14] and indeed the intensity of RAM11 immunostaining was much lower in these areas. The vast majority of PCNA-positive cells in the basal and suprabasal layers represent transit amplifying cells. This suggests that the expression of the studied antigen is associated with a transient differentiation event occurring in the "late" transit amplifying cells before they become postmitotic differentiating cells and migrate to higher layers of the epithelium.

Since the observed spatiotemporal pattern of RAM11 immunostaining in regenerating epithelium does not show intact edge to leading edge continuity, this expression does not strictly follow the sequence of events during epithelial repair. It is puzzling but can be related to the presence/absence of factors produced by various cells participating in wound healing, and possibly promoting or suppressing RAM11 expression in basal cells.
In conclusion, expression of the antigen recognized by RAM11 antibody in regenerating rabbit oral epithelium begins during maturation of the basal cells. In addition, it seems not to be associated with either melanocytes or antigen presenting cells (Langerhans cells). Our results also indicate that RAM11 can be regarded as basal cell marker only in mature stratified squamous epithelia.

\section{References}

[ 1] Tsukada T, Rosenfeld M, Ross R, Gown AM. Immunocytochemical analysis of cellular components in atherosclerotic lesions. Use of monoclonal anibodies with the Watanabe and fat-fed rabbit. Arteriosclerosis. 1986;6:601-613.

[2] Malinauskas RA, Herrmann RA, Truskey GA. The distribution of intimal white blood cells in the normal rabbit aorta. Atherosclerosis. 1995;115:147-163.

[3] Kimura H, Spee C, Sakamoto T et al. Cellular response in subretinal neovascularization induced by bFGF-impregnated microspheres. Invest Ophthalmol Vis Sci. 1999;40:524-528.

[4] Misao Y, Takemura G, Arai M et al. Importance of recruitment of bone marrow-derived CXCR4+ cells in post-infarct cardiac repair mediated by G-CSF. Cardiovasc Res. 2006;71: 455-465.

[ 5] St Pierre Schneider B, Brickson S, Corr DT, Best T. CD11b+ neutrophils predominate over RAM11+ macrophages in stretch-injured muscle. Muscle Nerve. 2002;25:837-844.

[ 6] Lis GJ, Litwin JA, Furgał-Borzych A et al. Macrophage-specific RAM11 monoclonal antibody cross-reacts wiyh basal cells of stratified squamous epithelia. Folia Histochem Cytobiol. 2007;45:229-232.

[7] Lavker RM, Sun TT. Heterogeneity in epidermal basal keratinocytes: morphological and functional correlations. Science 1982;215:1239-1241.

[8] Purkis PE, Steel JB, Mackenzie IC et al. Antibody markers of basal cells in complex epithelia. J Cell Sci. 1990;97:39-50.

[9] Kaur P, Li A. Adhesive properties of human basal epidermal cells: an analysis of keratinocyte stem cells, transit amplifying cells, and postmitotic differentiating cells. $J$ Invest Dermatol. 2000;114:413-420.

[10] Watt FM. Stem cell fate and pattering in mammalian epidermis. Curr Opin Genet Dev. 2001;11:410-417.

[11] Laplante AF, Moulin V, Auger FA et al. Expression of heat shock proteins in mouse skin during wound healing. J Histochem Cytochem. 1998;46:1291-1301.

Submitted: 8 July, 2009 Accepted after reviews: 19 October, 2009 